Ferdi Cakici

Omer Faruk Sukas

Omer Kemal Kinaci

Ahmet Dursun Alkan

http://dx.doi.org/10.21278/brod68203

ISSN 0007-215X

eISSN 1845-5859

\title{
PREDICTION OF THE VERTICAL MOTIONS OF THE DTMB 5415 SHIP USING DIFFERENT NUMERICAL APPROACHES
}

\author{
UDC 629.5.015.2:629.5.017.2:519.62
}

Original scientific paper

\begin{abstract}
Summary
Recent developments in Computational Fluid Dynamics (CFD) enabled common access for researchers and thus offers solutions to various complex problems in many different fields. With this motivation, in this study, two kinds of numerical methods were employed to investigate the vertical motions in variable regular waves. While the potential method is commonly known as linear "strip theory", the viscous approach is (the state of the art) named as URANS (Unsteady Reynolds Averaged Navier Stokes) solver which has a fully non-linear base. The DTMB 5415 ship model form was selected for a series of computational work. A numerical study was carried out to understand the seakeeping behaviour of the displacement hull for the stationary case $F n=0$ and a high speed case $F n=0.41$. The RAO (Response Amplitude Operator) graphs for the coupled heave - pitch motions and the ship's vertical accelerations were generated for five encounter frequencies. The numerical results obtained were validated with the existing experimental data and comparisons were made between the two numerical approaches with the help of RAO graphs. The obtained results showed that the limitations of the strip theory pose a handicap as the assumptions involved in the theory narrow down its application. The nonlinear viscous URANS approach tends to be a better option returning closer results to experiments in a wide Froude number range but on the other hand it does not possess the practicality of the strip theory.
\end{abstract}

Key words: Seakeeping; CFD, Strip Theory; URANS, ship motions;

\section{Introduction}

Investigation of ship motions in waves is one of the most challenging topics in the field of hydrodynamics. The difficulties arise from the nature of seakeeping calculations due to waves playing an important role on the ship's response. Viscous flows are highly nonlinear and the Reynolds numbers covered in ship motions are usually high which renders turbulent effects to be unavoidable. Gravitation complicates the problem even more because waves in nature are not in sinusoidal form as in regular waves. Additionally, the complex geometries of ship hulls majorly affect the restoring terms in the equations of motion. Linear theory regards the restoring terms as constant and this approach fails especially at higher wave amplitudes. 
Speaking in terms of a displacement hull, conducting seakeeping experiments are difficult and costly compared to experiments dealing with the resistance characteristics solely. The main reason is that measured forces and moments are time-dependent in seakeeping experiments compared to a (usually) fixed hull in resistance experiments. Some other challenges are listed below:

- There are difficulties to measure the amplitude of the waves reaching the ship. Also, the amplitudes of the generated waves are subject to change between the time of release of the wave and the time the ship encounters them.

- Radius of gyration of the model is difficult to obtain with enough level of accuracy.

Due to these difficulties in seakeeping experiments, numerical analysis methods may be used to support experiments utilizing its flow visualization abilities or even at some cases may be used as a substitute for experiments. Ship motion experiments are generally used to validate numerical approaches in the academic world [1].

Interest in seakeeping calculations started after 1940s and chronologically after wave resistance calculations using potential theory. The deficiency in computer technology by then hindered new computational methods to arise. Therefore, computation of ship motions was limited with linear theory until the end of 1970s. The most popular and widely known method was strip theory which still has grounds today. The ship geometry is divided by transversal finite sections for which the wave induced forces/moments and hydrodynamic reaction forces/moments (added mass and damping) are estimated. The results from every section are numerically integrated and any interaction between the sections is neglected. The wave induced excitation forces are estimated from the incident wave potential and the added mass and damping coefficients are derived from the local orbital velocities and accelerations assuming that the wave length is large in comparison with the sectional beam [2]. This approach is still not expired and it is practical to use in conceptual design stage of a ship. On the other hand, URANS calculations are recently used as a very good alternative to the linear theory with the development of the computer technology. URANS is especially recommended in cases where the experimental facilities are insufficient to respond or very expensive to set up.

URANS equations which are discretized with the Finite Volume Method are introduced to obtain 6DOF motion of a ship in regular and irregular waves. The inclusion of viscosity with various turbulence models in this approach increases the robustness of the method and its applicability in many cases. Some examples adopting URANS for ship motion calculations are given in references $[3,4]$.

Ursell solved the linear boundary value problem of a circular cylinder oscillating on the free surface in 1949 [5, 6]. The velocity potential is represented as a sum of an infinite set of multipoles satisfying the free surface boundary condition and each being multiplied by a coefficient to satisfy the body boundary condition. His studies formed a basis for the theory of ship motions. The first studies on seakeeping calculations were limited with 1DOF motion of a body in a fluid due to difficulties in determining the effects of hydrodynamic coefficients on each section. The theory assumes that ship motions are linearized and incident wave and body both oscillate with the same frequency. Added mass and damping terms are determined and integrated along the ship. Tasai combined Lewis' conformal mapping transformation with Ursell's method to generate a solution for more realistic ship sections [7, 8, and 9]. The method transforms the sections of a ship to a circle using a scale factor and two mapping coefficients. Ships having round bilge were correctly represented by his method. However, his method was insufficient to generate good results for SWATH ships or ships having transom sterns and bulbous bows. Frank has worked on this problem and developed a close-fit method [10]. 
Frank's method was better in representing arbitrary ship sections. The solutions generated by his method are valid for arbitrary cross sections and the velocity potential is represented by a distribution of sources on the mean submerged cross section. The method adopts Green function satisfying the linear free surface boundary condition to represent the velocity potential. The density of the sources is an unknown function to be determined from integral equations obtained by applying the body boundary condition [10]. Salvesen et al. presented the original strip theory by using 2-D hydrodynamic coefficients which are obtained by Frank's method [2]. With the strip theory, it was possible to get transfer functions (TF) for vertical motions of any 3-D ship form in regular waves. Strip theory is currently a common method to perform seakeeping analyses of a ship and there are many studies implementing the theory. It is proved in many studies that the strip theory is best in low Froude numbers and slender bodies but loses its effectiveness as the Froude number and beam/length ratio increases.

The nonlinear approach was used by many researchers to find the hydrodynamic coefficients regarding the added mass and the damping. One of those studies was the work of Querard et al. and they dealt with the computations of added mass and damping of 2D sections [11]. Calculations were done for a very wide range of frequency spectrum. The main focus of their work was to make a comparison with the results obtained by the potential theory, therefore, the motions of the sections that they selected were rather low. For this reason, Querard's method is more accurate compared with potential methods but still deficient due to low motion amplitude. Beck and Reed advises that the best option to solve for maritime problems are 3D URANS methods [12]. Sato et al. studied on coupled vertical motions for Wigley and Series 60 hull forms at head waves. They compared their results with experimental data. They concluded that CFD analyses are in good accordance with experiments for the Wigley hull but not Series 60 hull form [13]. Weymouth et al. also carried out seakeeping simulations by implementing URANS. Their results are compatible with experiments and they advised the best methods to be implemented in a wide Froude number range [14]. However, in this study the motions are investigated only for a low wave slope range. High wave slope ranges are investigated by Deng et al. and they investigated the vertical motions of a benchmark container ship form. Their study also included numerical uncertainty analyses [15]. Bhushan et al. performed seakeeping analyses for both the model and the full scale of DTMB 5415 ship hull. They also predicted manoeuvring derivatives for full scale hull [16]. Simonsen et al. prepared a comprehensive study by using URANS for many different types of ships for seakeeping calculations $[17,18]$. Wilson et al. carried out CFD simulations to obtain TF's of vertical responses of the S-175 ship in regular head waves [19].

In this study, Salvesen's strip theory was used to draw RAO graph of the DTMB 5415 destroyer model for coupled heave - pitch motions and CoG vertical acceleration. An in-house code developed by Sarioz et al. [20] was used for Strip Theory calculations. Finally, FVM which enables to discretize the URANS equations was used to obtain the RAO response of the hull to assess seakeeping abilities of the DTMB 5415 displacement hull. The commercial CFD software package Star-CCM+ was used to discretize the URANS equations by implementing finite volume method. The resistance characteristics of the ship in calm water was already calculated to assess the validation of the numerical approach with respect to experimental results. As it is indicated in the paper [21], $\mathrm{C}_{\mathrm{T}}$ was predicted with high level of accuracy. The present study under predicted $\mathrm{C}_{\mathrm{T}}$ at around $1 \%$ as compared with the experimental data.

This study aims to compare and contrast different numerical techniques and take advantage of their benefits when obtaining the vertical motions and accelerations in head waves for a high speed displacement ship. Large amplitude waves were also investigated and the results were presented in standard tabular format. 
Ferdi Cakici, Omer Faruk Sukas, Omer Kemal Kinaci, Ahmet Dursun Alkan
Prediction of the vertical motions of DTMB 5415

Ship Using Different Numerical Approaches

\section{Geometric Features and Physical Conditions}

In this study, a 1/24.83 scaled model of the DTMB 5415 hull given by Fig. 1 was used for numerical simulations. The experimental results were given in the reference report [22]. The main particulars of the model hull were given in Table 1 . All values were presented for the static case. The numerical simulations were performed for the bare hull case only without any appendages.

Table 1 Main particulars of the model

\begin{tabular}{|c|c|c|}
\hline Main parameters & Units & Value \\
\hline LwL & $\mathrm{m}$ & 5.720 \\
\hline BwL & $\mathrm{m}$ & 0.768 \\
\hline $\mathbf{T}$ & $\mathrm{m}$ & 0.248 \\
\hline LCG (from aft) & $\mathrm{m}$ & 2.924 \\
\hline S (Wetted surface area) & $\mathrm{m}^{2}$ & 4.786 \\
\hline VCG (from & $\mathrm{m}$ & 0.195 \\
\hline Displacement & $\mathrm{kg}$ & 549.0 \\
\hline $\mathbf{I}_{\mathbf{y y}}$ & $\mathrm{kg} \mathrm{\textrm {m } ^ { 2 }}$ & 1123.2 \\
\hline V & $\mathrm{m} / \mathrm{s}$ & $0.0-3.07$ \\
\hline Fn & - & $0.0-0.41$ \\
\hline
\end{tabular}

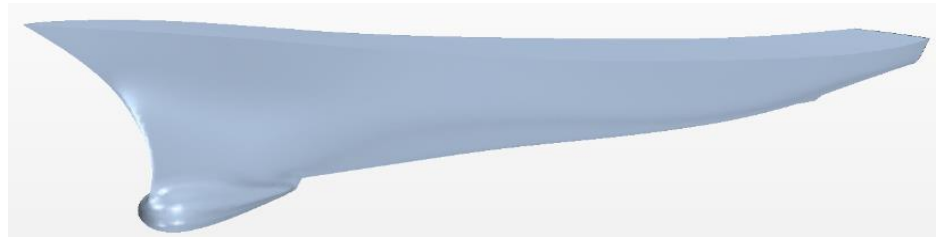

Fig. 13 D representation of the DTMB 5415 INSEAN Model

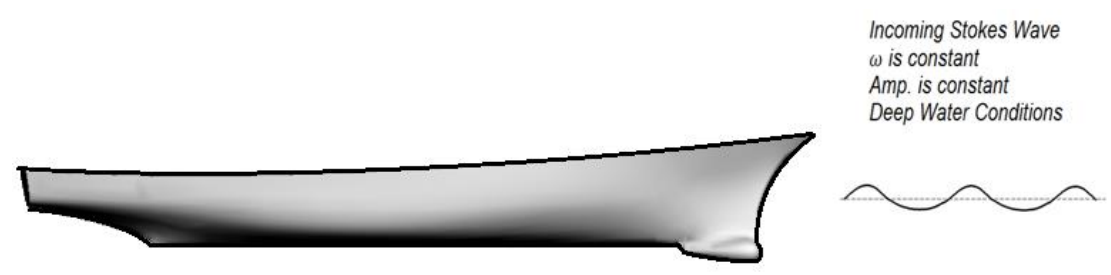

Fig. 2 Schematic physics

An Earth-fixed Cartesian coordinate system $x y z$ was selected for the solution domain. The $x y$ plane represented the calm free water surface and $z$ was defined as the vertical axis. The model was allowed to move in the positive $x$ direction with $2 D O F$ including heave and pitch motions only, as indicated in Fig. 2. A new local coordinate system was created for the ship to obtain 2DOF motion. The URANS and strip theory calculations were performed at five different encounter frequencies and two different velocities as shown in Table 2. Calculations were carried out with strip theory for $F n=0$ and $F n=0.41$ and with URANS for $F n=0.41$ only. All calculations were performed for five encounter frequencies at head waves.

Encounter frequency is defined as

$$
\omega_{e}=\omega+\left(\frac{\omega^{2}}{g V}\right)
$$

for head seas where $g$ denotes gravity, $\omega$ denotes frequency of the wave, $V$ denotes velocity of the ship in Equation (1). Small amplitude waves $(A * k=0.025)$ were selected for numerical simulations to be consistent with the experimental procedure [22] where $A$ denotes the wave amplitude and $k$ denotes the wave number. 
Table 2 Defined Cases for strip theory and URANS Calculations

\begin{tabular}{|c|c|c|c|c|c|c|c|}
\hline $\begin{array}{c}\text { Case } \\
\text { no. }\end{array}$ & Methods & Fn (-) & $A * k$ & $\begin{array}{c}\omega \\
(\mathrm{rad} / \mathrm{s})\end{array}$ & $\lambda / \mathbf{L}_{\mathrm{wl}}(-)$ & $\mathbf{H} / \lambda(-)$ & $\begin{array}{c}\text { Time step } \\
\text { size (s) }\end{array}$ \\
\hline 1 & \multirow{5}{*}{$\begin{array}{c}\text { Strip } \\
\text { Theory }\end{array}$} & \multirow{5}{*}{0.00} & \multirow{5}{*}{ N/A } & 2.357 & 1.939 & \multirow{5}{*}{ N/A } & \multirow{5}{*}{ N/A } \\
\hline 2 & & & & 2.488 & 1.740 & & \\
\hline 3 & & & & 2.750 & 1.425 & & \\
\hline 4 & & & & 2.946 & 1.241 & & \\
\hline 5 & & & & 3.142 & 1.091 & & \\
\hline 6 & \multirow{5}{*}{$\begin{array}{c}\text { Strip } \\
\text { Theory }\end{array}$} & \multirow{5}{*}{0.41} & 0.025 & 2.357 & 1.939 & $1 / 125$ & $\mathrm{~T}_{\mathrm{e}} / 2^{9}$ \\
\hline 7 & & & 0.025 & 2.488 & 1.740 & $1 / 125$ & $\mathrm{~T}_{\mathrm{e}} / 2^{9}$ \\
\hline 8 & & & 0.025 & 2.750 & 1.425 & $1 / 125$ & $\mathrm{~T}_{\mathrm{e}} / 2^{9}$ \\
\hline 9 & & & 0.025 & 2.946 & 1.241 & $1 / 125$ & $\mathrm{~T}_{\mathrm{e}} / 2^{9}$ \\
\hline 10 & & & 0.025 & 3.142 & 1.091 & $1 / 125$ & $\mathrm{~T}_{\mathrm{e}} / 2^{9}$ \\
\hline 11 & URANS & 0.41 & 0.1 & 2.357 & 1.939 & $1 / 31.25$ & $\mathrm{~T}_{\mathrm{e}} / 2^{9}$ \\
\hline
\end{tabular}

\section{URANS Equations and Modeling}

The averaged continuity and momentum equations can be written for incompressible flow in cartesian coordinates and tensor form as indicated Equation (2) and (3):

$$
\begin{aligned}
& \frac{\delta U_{i}}{\delta x_{i}}=0 \\
& \rho\left(\frac{\delta U_{i}}{\delta t}+U_{j} \frac{\delta U_{i}}{\delta x_{i}}\right)=-\frac{\delta P}{\delta x_{i}}+\frac{\delta \tau}{\delta x_{j}}-\frac{\delta\left(\rho \overline{u_{i}^{\prime} u_{j}^{\prime}}\right)}{\delta x_{j}}+F_{i}
\end{aligned}
$$

where $\tau_{\mathrm{ij}}$ are the mean viscous stress tensor components and shown in Equation (4).

$$
\tau=\tau_{i j}=\mu\left(\frac{\delta U_{i}}{\delta x_{j}}+\frac{\delta U_{i}}{\delta x_{i}}\right)
$$

In this paper, two equation $k-\varepsilon$ turbulence model was used to include the effects of viscosity as it is considered to be one of the most commonly used turbulence model for industrial applications [23]. It is also cheaper in terms of computer memory compared to the $k$ $\omega S S T$ model which requires higher CPU time $[4,23]$. The employed solver uses a finite volume method which discretizes the Navier-Stokes (N-S) equations for numerical model of fluid flow. Segregated flow model was used in the URANS solver and convection terms in the URANS equations were discretized by applying second order upwind scheme. In the analyses, the URANS solver runs a predictor-corrector SIMPLE-type algorithm between the continuity and momentum equations. A first-order temporal scheme was applied to discretize the unsteady term in the N-S equations. Volume of Fluid (VOF) model was used to represent the free surface. In this model, computations were performed for water and air phases. Due to the mesh structure and the number of elements having great importance in capturing the free surface deformations, some refinements were defined close to the free surface to accurately predict VOF wave profiles. Second order convection scheme was used to present results calculated by VOF more precisely. All the analyses were performed in deep water conditions.

The flow within the boundary layer has to be solved correctly to accurately calculate boundary layer dynamics. Therefore, $y^{+}$values on the hull surface should stay within the limits for the $k-\varepsilon$ turbulence model. The $y^{+}$values on the hull surface were around 60 as it can be seen from Fig. 3. This value is considered to be suitable since it remains between the recommended ranges 30-300 for the selected turbulence model [24]. 


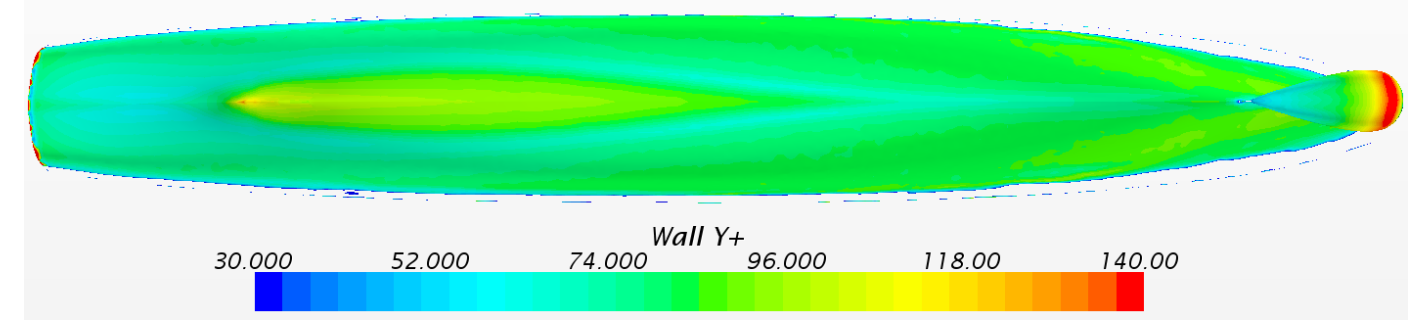

Fig. 3 Averaged $\mathrm{y}^{+}$values on the hull surface

DFBI (Dynamic Fluid Body Interaction) module in the commercial software STAR $\mathrm{CCM}+$ was used for the motion of body and the vessel is set free to heave and pitch. The 2DOF motion of the body was obtained by calculating the velocity and pressure field in the fluid domain. For this purpose, linear and angular momentum equations, given in Equation (5) and (6) respectively, were solved:

$$
\begin{aligned}
& \sum \vec{F}=m \vec{a} \\
& \sum \overrightarrow{M_{G}}=I_{G} \overrightarrow{\alpha_{a}}+\vec{\omega} \times I_{G} \vec{\omega}
\end{aligned}
$$

where $F$ denotes the total force, $m$ the mass, $a$ the acceleration, $M_{G}$ the moment taken from the center of gravity, $I_{G}$ the inertial mass moment, $\alpha_{\mathrm{a}}$ the angular acceleration and $\omega$ is the angular speed.

\subsection{Selection of Time Step Size}

In this study, implicit method was used due to computational limitations. Explicit method requires higher computer memory due to the relatively larger fluid domain demanded by the flow physics of ship hydrodynamic problems. In the explicit method, CFL condition has to be satisfied for greater accuracy and the stability of the method. In the unsteady implicit problems, the restriction imposed by the CFL condition is not an issue anymore which relaxes the computer in terms of required memory.

Time step size was selected to be $1 / 2^{9}$ of $T_{e}$ for seakeeping analyses which is considered to be more accurate than the value recommended by ITTC [25]. Here $T_{e}$ denotes encounter period. It is believed that the smaller time step size chosen in this study better captures the physics. The variation of time step size and the generated results are given in the CFD verification and validation section. Analyzing Table 6, it may be said that there is still room for improvement in the generated results although the selected time step sizes were much lesser than the one recommended by the ITTC.

\subsection{Computational Domain and Boundary Conditions}

The initial and boundary conditions must be suitable for all numerical and analytical problems in order to have a well posed problem. These conditions must be defined in accordance with the characteristics of the flow. In this study, the computational domain was created in order to simulate the seakeeping behavior of the naval surface combatant in regular waves. The rigid body motion approach was used for representing coupled heave and pitch motions of the ship.

By defining symmetry plane for the centerline of the ship, only half of the body was modeled reducing the domain size and computational time. The boundary conditions are illustrated in Fig. 4. 


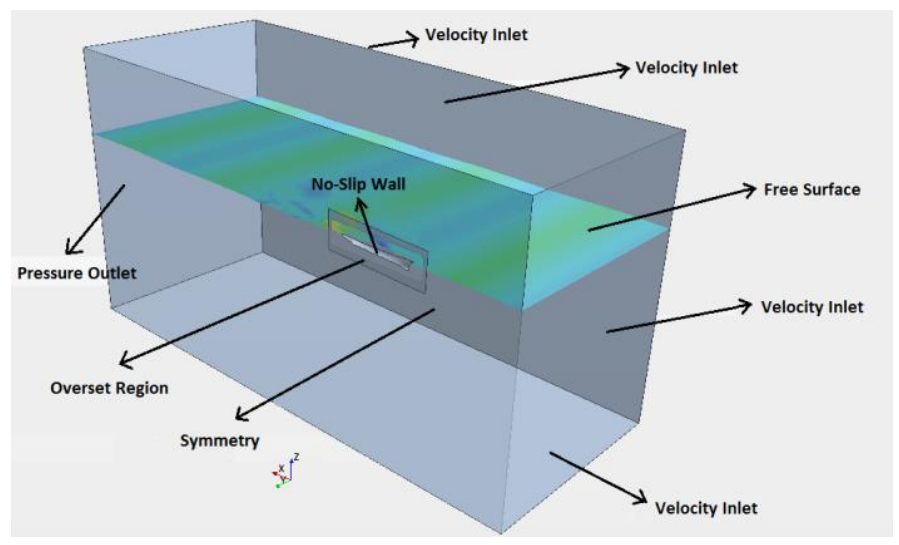

Fig. 4 Boundary conditions in the computational fluid domain.

As it is seen in Fig. 4, the top, bottom and side boundaries were modeled as velocity inlet to avoid formation of boundary layers that would form near these boundaries. This also helps in accelerating the numerical simulation. The $5^{\text {th }}$-order Stokes waves were used to represent the wavy environment for all URANS analyses. This wave profile was selected due to the fact that it is more like a real wave than the one generated by the first order method [4].

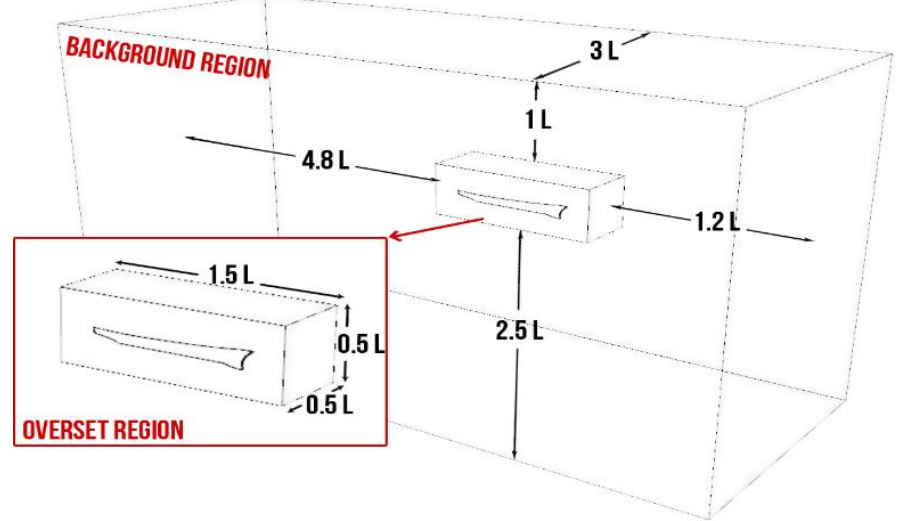

Fig. 5 Computational Domain Size

\subsection{Mesh Structure}

The trimmer mesh module in STAR CCM+ ensures a secured and efficient method to generate high quality mesh even for complex bodies [24]. The trimmer mesh mostly contains hexahedral elements having minimal cell skewness. This tool also provides the following:

-A prism layer mesh for high Reynolds numbers,

-Automatic local refinement in the cells,

-Creating volumetric controls with particular shapes

As it can be seen from Figure 6, the grid system applied in CFD calculations consisted of two blocks, overset and background regions. The background grids were fixed to global coordinate system but the overset grids were moving with the body. Overset mesh, which is considered to have great flexibility for bodies moving inside the fluid, was used for calculation of ship motions. This grid system, which is embedded in the background mesh enclosing the certain zone of domain, was used to represent the motion of the hull and there is an "overlap" zone that encompasses the overset region. The information is passed through the overlap block between overset and background regions. With the overset grid system, any mesh modification or deformation is not necessary which provides great flexibility over the other standard meshing techniques. 
Ferdi Cakici, Omer Faruk Sukas, Omer Kemal Kinaci, Ahmet Dursun Alkan
Prediction of the vertical motions of DTMB 5415

Ship Using Different Numerical Approaches

The computational domain extended for $1.2 \mathrm{~L}$ in front of the overset region, $4.8 \mathrm{~L}$ behind the overset region, and $3 \mathrm{~L}$ to the side and $2.5 \mathrm{~L}$ under the boundaries of overset region as it is seen from Fig.5. The air region was $1 \mathrm{~L}$ above the overset region. The mesh was then refined at five regions; overset region, overlap region, vicinity of the hull, around free surface and Kelvin wake region where wave deformation is significant. Refinement blocks were also added near the ship's bow and stern regions in order to capture the pitch motion well. Three different unstructured hexahedral mesh systems were used to calculate numerical uncertainty. The number of elements are given in Table 3. Fig. 6 shows the coarse mesh and refinements at critical zones. Numbers on Fig. 6 indicates the volumetric controls and they are:

1. Background region

2. Overset region

3. Overlap region

4. Free surface refinements

5. Bow\&Stern refinements

Table 3 Mesh Numbers for $F n=0.41$

\begin{tabular}{|c|c|c|c|}
\hline Case no. & Coarse Mesh & Medium Mesh & Fine Mesh \\
\hline \multirow{4}{*}{ from 6 to 11 } & Background Mesh & Background Mesh & Background Mesh \\
\cline { 2 - 4 } & $540 \mathrm{k}$ & $\mathbf{1 0 8 0 k}$ & $2160 \mathrm{k}$ \\
\cline { 2 - 4 } & Overset Mesh & Overset Mesh & Overset Mesh \\
\cline { 2 - 4 } & $1080 \mathrm{k}$ & $\mathbf{2 1 6 0 k}$ & $4320 \mathrm{k}$ \\
\cline { 2 - 4 } & Total Mesh & Total Mesh & Total Mesh \\
& $1620 \mathrm{k}$ & $\mathbf{3 2 4 0 k}$ & $6480 \mathrm{k}$ \\
\hline
\end{tabular}

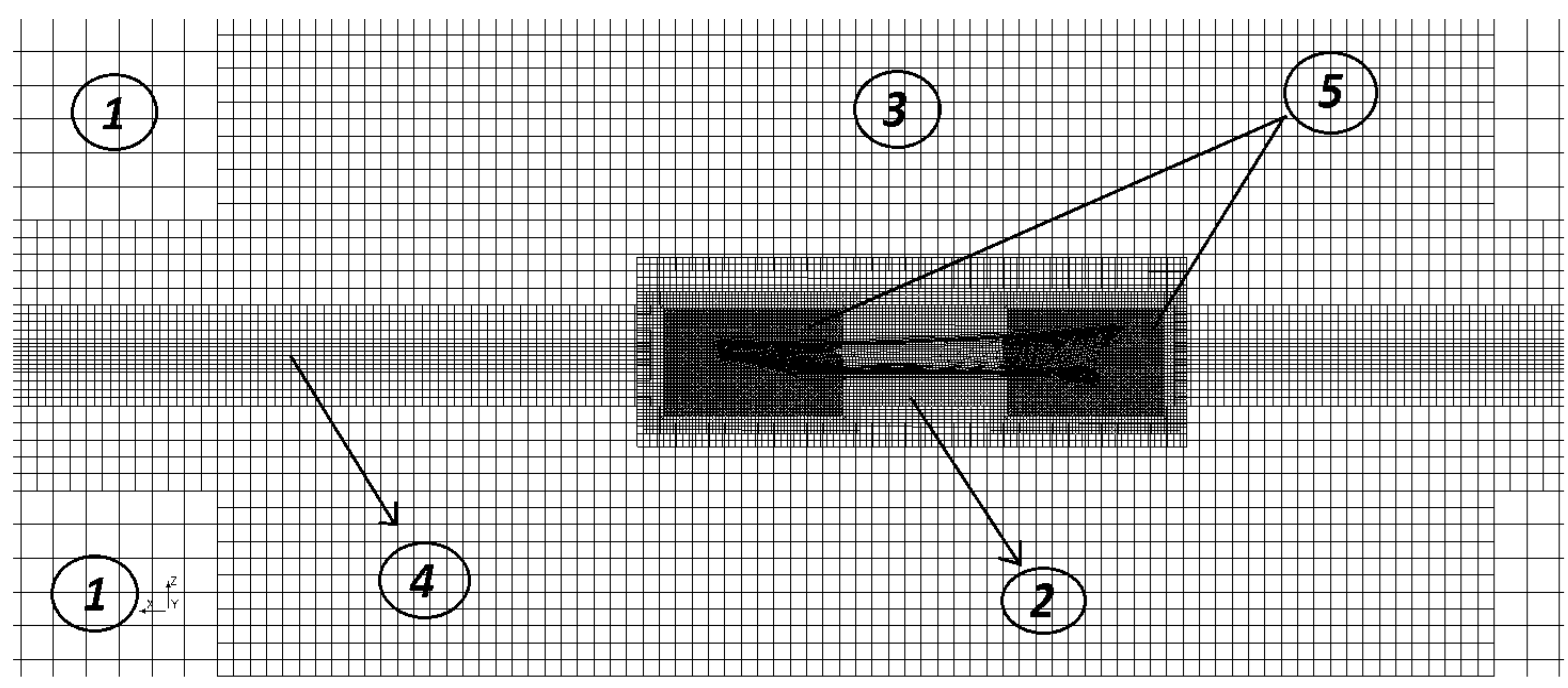

Fig.6. Mesh structure in the fluid domain 


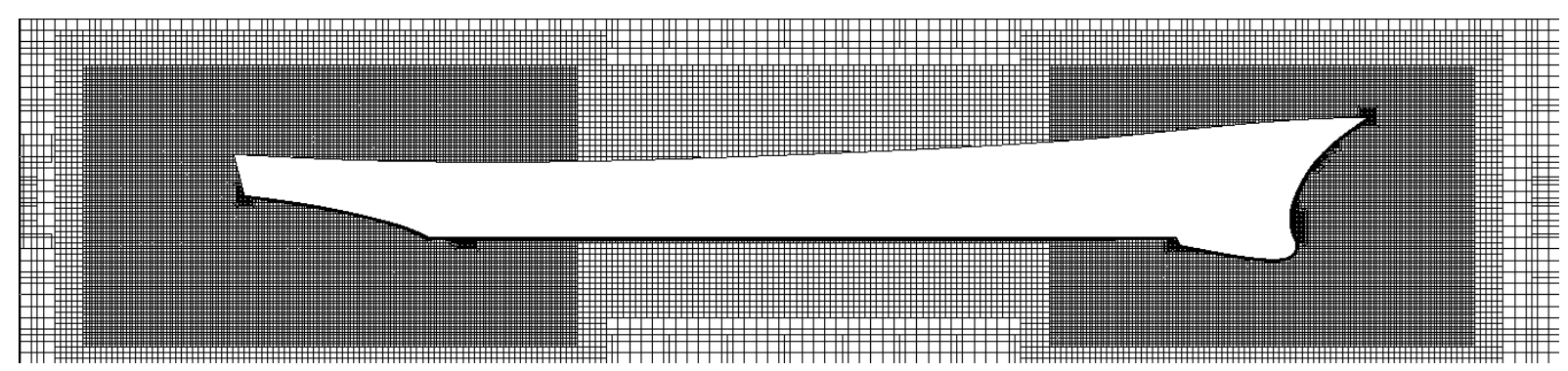

Fig. 7. Overset mesh structure around the ship

In overset grid implementation, there is no re-meshing process and the elements do not deform or modify. The ship is moving together with the grid system which makes it consistent in terms of element quality. Once a high-quality grid system is settled in the fluid domain, the numerical setup will use the same elements throughout the analysis. Fig. 7 shows the overset region around the ship.

\subsection{Fourier series (FS) Formulation}

Unsteady time histories of the analyzed motions, $\eta(t)$ can be represented by using FS as indicated in Equation (7).

$\eta(t)=\eta_{0}+\sum_{n=1}^{N} \eta_{n} \cos \left(\omega_{e} t+\beta_{n}\right)$

$n=1,2,3 \ldots$

In Equation (8) and (9), $\eta_{n}$ and $\beta_{n}$ denotes $\eta_{\text {th }}$ harmonic amplitude and phase angle respectively. These values can be calculated as follows by using $a_{n}$ and $b_{n}$ in Equation (10) and (11).

$\eta_{n}=\sqrt{a_{n}^{2}+b_{n}^{2}}$

$\beta_{n}=\arctan \left(\frac{b_{n}}{a_{n}}\right)$

$a_{n}=\frac{2}{T_{e}} \int_{0}^{T_{e}} \eta(t) \cos \left(\omega_{e} n t\right) d t$

$b_{n}=\frac{2}{T_{e}} \int_{0}^{T_{e}} \eta(t) \sin \left(\omega_{e} n t\right) d t$

In these equations $T_{e}$ refers to sampling time and it is the encounter period of the given signal. Vertical ship motions, heave and pitch in regular waves can be expressed in terms of RAO'S by the following statements given in Equation (12) and (13):

$$
\begin{aligned}
& R A O_{\text {Heave }}=\frac{\eta_{1 \text { Heave }}}{A} \\
& R A O_{\text {Pitch }}=\frac{\eta_{1 \text { Pitch }}}{A k}
\end{aligned}
$$


Ferdi Cakici, Omer Faruk Sukas, Omer Kemal Kinaci, Ahmet Dursun Alkan
Prediction of the vertical motions of DTMB 5415

Ship Using Different Numerical Approaches

\section{CFD Verification and Validation}

Verification and validation $(\mathrm{V} \& \mathrm{~V})$ test $[26,27]$ was made for computational studies involved in this work to assess numerical uncertainties which is a combination of iterative, grid and time step uncertainties $\left(U_{I}, U_{G}, U_{T}\right.$ respectively). Numerical simulation uncertainty $U_{S N}$ is given in Equation (14):

$$
U_{S N}=\sqrt{U_{I}^{2}+U_{G}^{2}+U_{T}^{2}}
$$

The V\&V study was made for $F n=0.41$ and at a wave frequency of $\omega=2.357 \mathrm{rad} / \mathrm{s}$. Iterative uncertainties in all trials were found to be very low when compared with grid and time step uncertainties; therefore, it was assumed that $U_{I}=0$. The element numbers (of the grids $G 1$, G2, G3 and G4) and time step sizes (T1,T2, T3 and T4) are given in Table 4.

Table 4 Element numbers and time step sizes involved in the uncertainty study.

\begin{tabular}{|c|c|c|c|c|}
\hline & G4 & G3 & G2 & G1 \\
\hline Element no. & 750,000 & $1,500,000$ & $3,000,000$ & $6,000,000$ \\
\hline
\end{tabular}

\begin{tabular}{|c|c|c|c|c|}
\hline & T4 & T3 & T2 & T1 \\
\hline Time Step Size & $\mathrm{T}_{\mathrm{e}} / 2^{7}$ & $\mathrm{~T}_{\mathrm{e}} / 2^{8}$ & $\mathrm{~T}_{\mathrm{e}} / 2^{9}$ & $\mathrm{~T}_{\mathrm{e}} / 2^{10}$ \\
\hline
\end{tabular}

Here, $T_{e}$ is the encounter period. The results of the grid and time step convergence studies are given in Tables 5 and 6 . The number of elements in each grid were twice the previous grid as can be seen in Table 4 and therefore the refinement ratio in grid convergence study is equal to $r_{G}=\sqrt[3]{2}=1.2599$. The time steps were increased with a refinement ratio of 2 , starting from $T_{e} / 2^{7}$. The grid uncertainties as a percentage of grid 3 (G3,1.5M elements) for heave and pitch were $3.44 \%$ and $0.43 \%$ respectively as it is seen from Table 5 . The time step uncertainty as percentage of time step $2\left(T 2, T_{e} / 2^{9}\right)$ for heave and pitch were found to be $5.07 \%$ and $2.88 \%$ respectively as it is seen from Table 6 . The simulation numerical uncertainty for heave was around $6 \%$ while it was around 3\% for pitch as it is indicated in Table 7 . It should also be taken into notice from this table that although the numerical uncertainty in pitch is less than heave, the obtained result range lies outside the EFD (experimental fluid dynamics) result. However, this is not considered to be a major issue due to the grid convergence ratio $R_{G}$ (which is calculated for grid 2 and given as $R_{G 2}$ in table 5) having small values and achieving monotonic convergence.

Time step convergence study is given in table 6 . In that table, $r_{T}$ denotes the refinement ratio in time step size and $R_{T 3}$ denotes the time step convergence ratio calculated for $T 3$.

Table 5. Grid convergence study

\begin{tabular}{|l|l|l|l|l|l|l|l|l|}
\hline & G4 & G3 & G2 & G1 & rG & R $_{\mathbf{G}}$ & $\mathbf{U}_{\mathbf{G}}(\mathbf{\% G} \mathbf{3})$ & EFD \\
\hline Heave & 1.0177 & 1.0289 & 1.0309 & 1.0505 & 1.2599 & 0.184 & 3.44 & 1.0563 \\
\hline Pitch & 1.1072 & 1.1596 & 1.1613 & 1.1619 & 1.2599 & 0.325 & 0.43 & 1.2399 \\
\hline
\end{tabular}


Table 6. Time step convergence study

\begin{tabular}{|l|l|l|l|l|l|l|l|l|}
\hline & T4 & T3 & T2 & T1 & rT & $\mathbf{R}_{\text {T3 }}$ & $\mathbf{U}_{\mathbf{T}}\left(\mathbf{\% T}_{2}\right)$ & EFD \\
\hline Heave & 0.9100 & 0.9835 & 1.00289 & 1.0527 & 2 & 0.523 & 5.07 & 1.0563 \\
\hline Pitch & 0.9977 & 1.1081 & 1.1595 & 1.1792 & 2 & 0.383 & 2.88 & 1.2399 \\
\hline
\end{tabular}

Table 7. Validation of heave and pitch

\begin{tabular}{|c|c|c|c|c|}
\hline & Values & UsN $(\%)$ & Result range & EFD \\
\hline Heave & 1.0289 & 6.13 & $0.9659-1.0919$ & 1.0563 \\
\hline Pitch & 1.1596 & 2.91 & $1.1258-1.1934$ & 1.2399 \\
\hline
\end{tabular}

\section{Results and Discussions}

The presented results and discussion on vertical motions-acceleration in regular head waves of DTMB 5415 are shown in this section. Results are compared with experimental data of the same scale model in terms of seakeeping [22].

Heave and pitch RAO graphs for $F n=0$ and $F n=0.41$ which were obtained by applying strip theory and URANS are demonstrated in this part. Results generated by the strip theory (which are cases 1 through 5 at $F n=0$ ) are given in Fig. 7 and 8 in comparison with experiments As it is seen from Fig. 7 and 8, linear strip theory is convenient for both motions when $F n=0$ in regular waves. It can be seen that strip theory gives satisfactory RAO values in all frequency intervals. Due to strip theory providing accurate results in the case of a stationary ship in waves, there is no need to tackle the high computational cost of URANS solvers. This was actually an expected result as it is already known that strip theory is suitable to be implemented at low ship speeds.

As for $F n=0.41$ cases ( 6 through 10), time histories of the coupled heave and pitch motions were obtained using the medium grid (G3) for all cases. First harmonics were derived by applying FS for each case. Then RAO's for coupled heave and pitch motions were generated and compared with the experimental RAO's for $A^{*} k=0.025$. Figures 9 and 10 reveal the nondimensional heave amplitudes obtained experimentally, by strip theory and by URANS for $F n=0.41$. Over the entire frequency range, strip theory over predicted the heave response of the ship but generally the results were in good accordance with experiments as the general trends of the curve agreed. URANS predictions were closer to the experiments but it was unable to capture the experimental resonance frequency which was around $2.95 \mathrm{rad} / \mathrm{s}$. The pitch response of the hull calculated by URANS was generally in good agreement with experiments, given in Fig. 10. It may be said that strip theory generated results were also satisfactory although the calculated changes in the pitch response with respect to the changes in the wave frequency were more gradual. The results presented in Figures 9 and 10 are tabulated in Table 9. 
Ferdi Cakici, Omer Faruk Sukas, Omer Kemal Kinaci, Ahmet Dursun Alkan
Prediction of the vertical motions of DTMB 5415

Ship Using Different Numerical Approaches

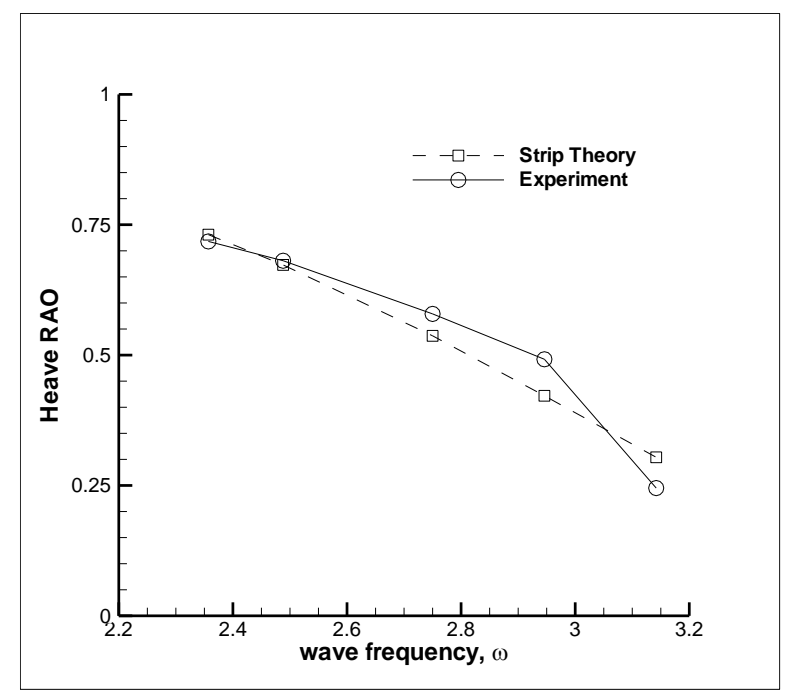

Fig. 7. Heave RAO for $\mathrm{Fn}=0$

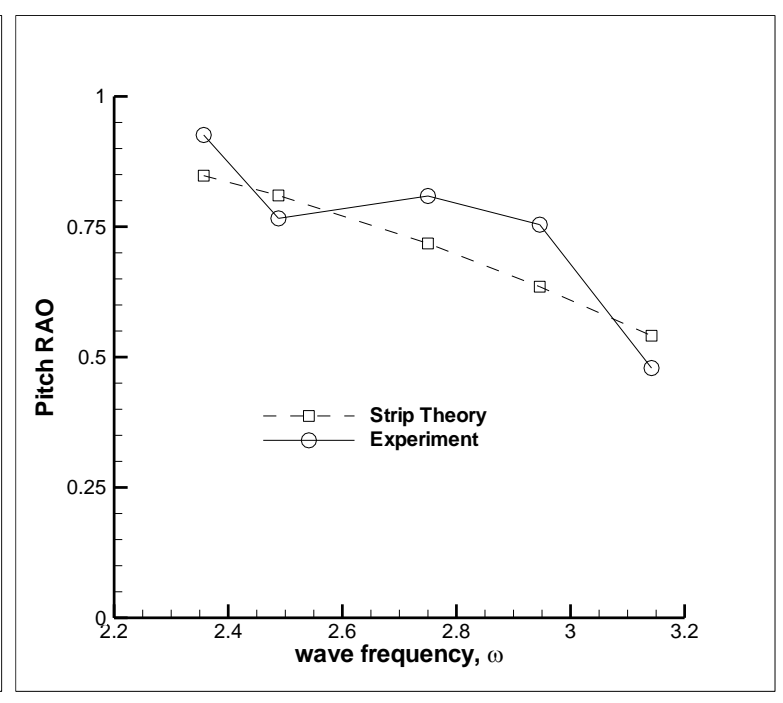

Fig. 8. Pitch RAO for $\mathrm{Fn}=0$

The case at $F n=0.41$ is considered as high speed for a displacement vessel. The reason why the nonlinear model implementing URANS returns better results compared to the linear strip theory at this speed is because the flow around the ship is highly turbulent and viscous effects are playing a more important role in the vessel's response in waves. Another simplification of the linear model is the absence of the interaction between transversal sections as strip theory takes into account the forces only acting at that specific section. Therefore, it is advised that this case should be investigated with non-linear tools especially when dealing with wave frequencies close to the resonance region where the vessel is making significant motions in the sea.

Results for case 11 were only derived by URANS as strip theory is unavailable at this wave amplitude. The experimental results are not available neither. The numerical simulations were made to understand the ship's response at higher wave amplitudes. The generated results for case 11 and its comparison with case 6 are given in Table 10. As expected the amplitudes of the motions were significantly larger in the case of the larger amplitude waves. As for speaking non-dimensional RAO, there was a slight reduction on the heave and pitch RAO's on large amplitude regular waves. However, presented results for the large amplitude waves at case 11 were not verified since experimental data do not exist.

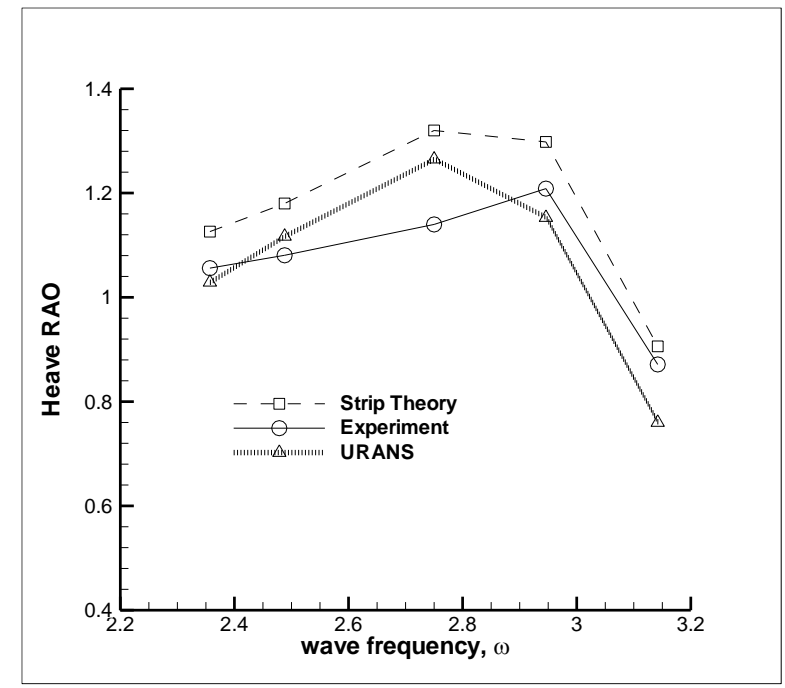

Fig.9. Heave RAO for $\mathrm{Fn}=0.41$

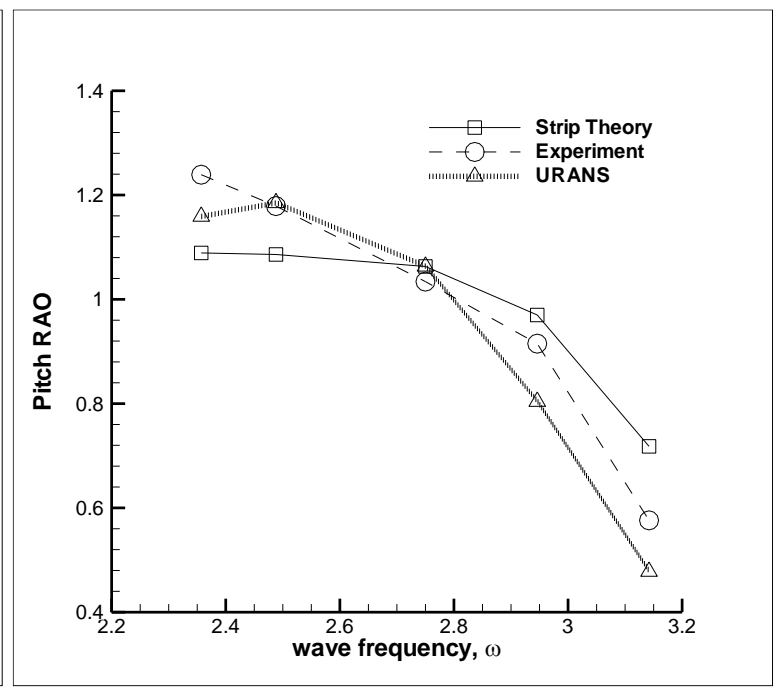

Fig. 10. Pitch RAO for $F n=0.41$ 
Table 9. RAO's for both heave and pitch motions at $F n=0.41$

\begin{tabular}{|c|c|c|c|c|c|c|}
\hline \multirow{2}{*}{$\begin{array}{c}\text { Case } \\
\text { No. }\end{array}$} & \multicolumn{3}{|c|}{ Heave RAO } & \multicolumn{3}{c|}{ Pitch RAO } \\
\cline { 2 - 7 } & URANS & Strip & EXP. & URANS & Strip Theory & EXP. \\
\hline Case 6 & 1.028 & 1.126 & 1.056 & 1.159 & 1.089 & 1.239 \\
\hline Case 7 & 1.117 & 1.18 & 1.080 & 1.185 & 1.086 & 1.178 \\
\hline Case 8 & 1.265 & 1.32 & 1.139 & 1.063 & 1.063 & 1.034 \\
\hline Case 9 & 1.153 & 1.298 & 1.208 & 0.804 & 0.97 & 0.915 \\
\hline Case 10 & 0.833 & 0.906 & 0.871 & 0.511 & 0.718 & 0.576 \\
\hline Case 11 & 1.018 & - & - & 1.091 & - & - \\
\hline
\end{tabular}

Vertical acceleration RAO was calculated only at the center of gravity, CoG, and given in non-dimensional form as given in Equation (19):

$$
R A O_{\text {Vert. Acce. }}=\frac{A c c e \times L_{W L}}{g \times A}
$$

RAOVert.Acce graphs which also corresponds to the heave accelerations are given in Figures 11 and 12 for $F n=0$ and $F n=0.41$ respectively for cases 0 through 10 . As it may be noticed from these figures, the velocity of the ship significantly increases the vertical accelerations occuring on the ship.

Due to the many assumptions the linear theory contains, strip theory is limited with low wave amplitudes. Therefore the results presented in Figures $7-10$ where $A^{*} k=0.025$, are in a range that strip theory can be used safely. Case 11 is a replica of case 6 where the wave frequencies and the wavelengths are equal and only the wave amplitudes are different. In case $11, A^{*} k$ is 0.1 and this high value was considered to be outside the scope of strip theory. The coupled heave and pitch motion values given in Table 10 for cases 6 and 11 showed that the ship motions are greater in higher wave amplitudes which nullifies the effectiveness of strip theory.

Table 10. Coupled heave and pitch motion values

\begin{tabular}{|c|c|c|c|}
\hline Case No. & Incident Wave & Heave Motion & Pitch Motion \\
\hline Case 6 & 0.044 & 0.0452 & 1.655 \\
\hline Case 11 & 0.176 & 0.1791 & 6.233 \\
\hline
\end{tabular}




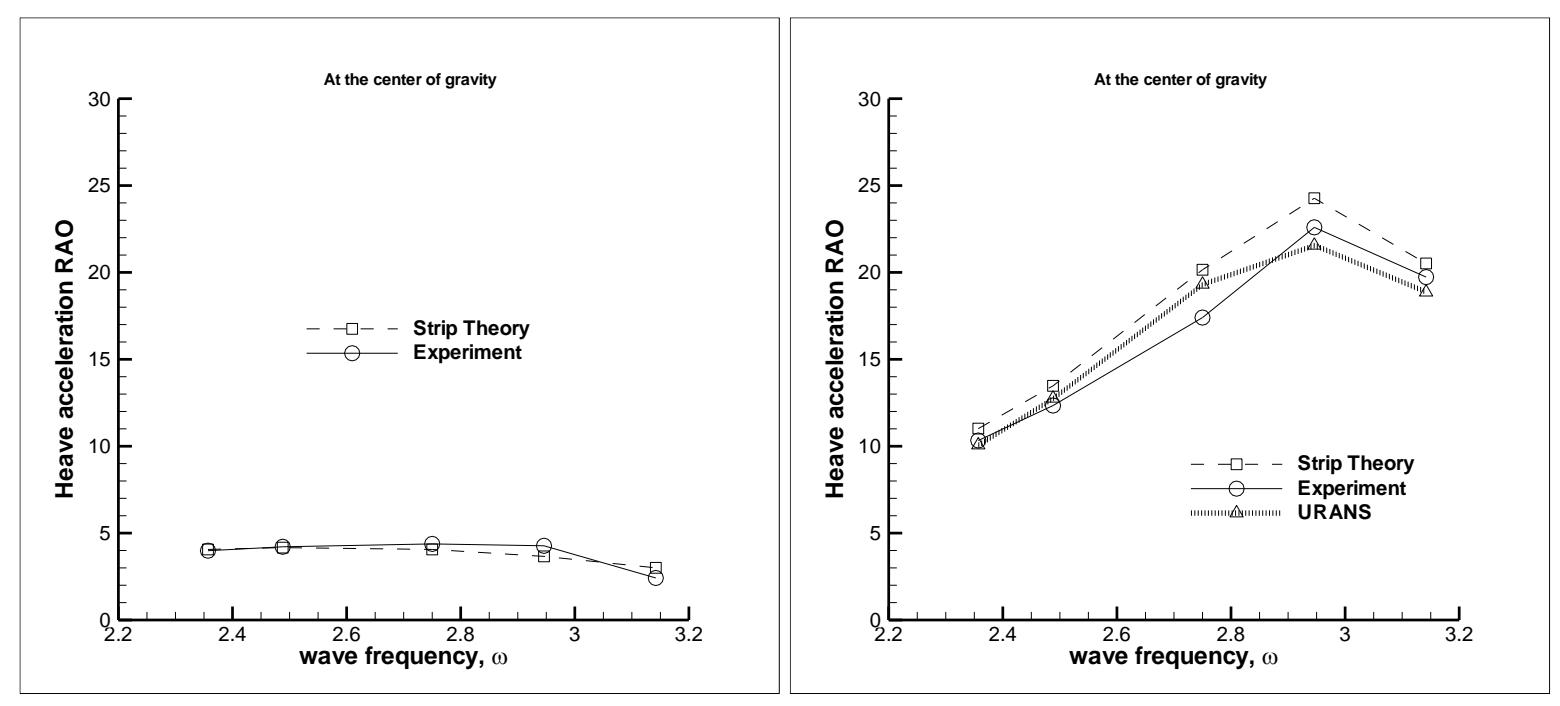

Fig.11. $\mathrm{CoG}$ acceleration RAO for $\mathrm{Fn}=0$

Fig.12. $\mathrm{CoG}$ acceleration RAO for $\mathrm{Fn}=0.41$

\section{Conclusions}

In the present paper, the coupled heave - pitch motions and heave accelerations on regular head waves for several cases were investigated with linear and nonlinear approaches. The linear approach implemented in this study was the widely used strip theory while for the nonlinear approach, URANS was adopted to solve the viscous flow around the ship. Strip theory has proven its worth in time while URANS is still being tested in the last few decades as the nonlinear models are getting more complex and accurate in time. Speaking in terms of motions of the hull in regular head waves, strip theory was used for stationary and high speed cases for small amplitudes. On the other hand, URANS was only used for the high speed case for small and large amplitude regular head waves to prove the capability of the nonlinear approach. For $F n=0.41$, covering the cases 6 through 11, the RAO graphs calculated by URANS showed a better agreement compared to those obtained by strip theory. A slight reduction on RAO value was observed on the heave and pitch RAO's in large amplitude regular head waves. It was also observed that the vertical acceleration levels significantly increased at $F n=0.41$ which is considered to be high speed for a displacement vessel.

As a conclusion, it is found out that the strip theory is best in low Froude numbers in terms of computational time and it loses its effectiveness as the Froude number increases. The limitation of the strip theory is also a drawback and restricts its application in many cases. The fully nonlinear viscous URANS approach is generally a better option returning closer results to experiments in a wide Froude number range however it does not possess the practicality of the strip theory.

\section{Acknowledgment}

This research has been supported by Yildiz Technical University Scientific Research Projects Coordination Department. Project No: 2015-10-01-KAP01. The first author was supported by ASELSAN Graduate Scholarship for Turkish Academicians. The first author would like to thank Prof. Kadir SARIOZ from Istanbul Technical University for his continuous support throughout his $\mathrm{PhD}$ period. 
Estimation of Vertical Motions of DTMB 5415

Ship Hull with a Fully Nonlinear Approach
Ferdi Cakici, Omer Faruk Sukas,

Omer Kemal Kinaci, Ahmet Dursun Alkan

\section{REFERENCES}

[1] Bertram V. Practical Ship Hydrodynamics. Butterworth-Heinemann. 2000.

[2] Salvesen,N. Tuck, O. and Faltinsen,O. Ship Motions and Sea Loads. The Society of Naval Architects and Marine Engineers.1970.

[3] Ozdemir, Y.H., Barlas B., Yilmaz T., Bayraktar S., Numerical and experimental study of turbulent free surface flow for a fast ship. Brodogradnja, 65(1), 39-54, 2014.

[4] Tezdoğan T., Demirel Y.K., Kellett P., Khorasanchi M., Incecik A., Turan O. Full-scale unsteady RANS CFD simulations of ship behavior and performance in head seas due to slow steaming. Ocean Engineering, 186-206. 2015. https://doi.org/10.1016/j.oceaneng.2015.01.011.

[5] Ursell, F. On the heaving motion of a circular cylinder in the surface of a fluid. Quart. J. Mech. Appl. Math, 2: 218-231. 1949. https://doi.org/10.1093/qjmam/2.2.218.

[6] Ursell, F. On the rolling motion of a circular cylinder in the surface of a fluid, Quart. J. Mech. Appl. Math., 2: 335-353. 1949. https://doi.org/10.1093/qjmam/2.3.335.

[7] Lewis, F.M. The inertia of water surrounding a vibrating ship, Transactions, Society of Naval Architects and Marine Engineers, 27: 1-20. 1929.

[8] Tasai,F. On the Damping Force and Added Mass of Ships Heaving and Pitching. Technical Report, Res. Ins. for Applied Mechanics, Kyushu University, Japan, Vol. VII, No 26. 1959.

[9] Tasai,F. Hydrodynamic Force and Moment Produced by Swaying and Rolling Oscillations of Cylinders on the Free Surface. Technical Report, Res. Ins. for Applied Mechanics, Kyushu University, Japan, Vol. IX, No 35. 1959.

[10] Frank, W. Oscillation of Cylinders in or Below the Free Surface of Deep Fluids, DTNSRDC Report No. 2375. 1967.

[11] Querard, A.B.G., Temarel, P. and Turnock, S.R. The hydrodynamics of ship-like sections in heave, sway and roll motions predicted using an unsteady Reynolds averaged Navier-Stokes method. Engineering for the Maritime Environment, 233. 2010.

[12] Beck, R.F. and Reed, A.M. 'Modern computational methods for ships in a seaway'. Transactions of the Society of Naval Architects and Marine Engineers, 109 pp.1-51.2001.

[13] Sato, Y., Miyata, H. and Sato, T. CFD simulation of 3-dimensional motion of a ship in waves: application to an advancing ship in regular heading waves. Journal of Marine Science and Technology, 4: 108-116. 1999. https://doi.org/10.1007/s007730050013.

[14] Weymouth, G.D., Wilson, R.V. and Stern, F.RANS computational fluid dynamics predictions of pitch and heave motion in head seas. Journal of Ship Research. 49(2): 80-97. 2005.

[15] Deng, G.B., Queutey, P. and Visonneau, M. Seakeeping prediction for a container ship with RANS computation, 22nd Chinese conference in hydrodynamics. 2009.

[16] Bhushan, S., Xing, T., Carrica, P. and Stern, F.Model- and full-scale URANS simulations of Athena resistance, powering, seakeeping, and 5415 maneuvering. Journal of Ship Research, 53 (4), pp.179-198. 2009.

[17] Simonsen, C.D., Otzen, J.F., Joncquez, S. and Stern, F. EFD and CFD for KCS heaving and pitching in regular head waves. Journal of Marine Science and Technology, 18 (4), pp.435-459. 2013. https://doi.org/10.1007/s00773-013-0219-0.

[18] Simonsen, C.D. and Stern, F. CFD simulation of KCS sailing in regular head waves. Gothenburg 2010A Workshop on Numerical Ship Hydrodynamics. Gothenburg. 2010.

[19] Wilson, R.V., Ji, L., Karman, S.L., Hyams, D.G., Sreenivas, K., Taylor, L.K. and Whitfield, D.L. Simulation of large amplitude ship motions for prediction of fluid-structure interaction. 27th Symposium on Naval Hydrodynamics. Seoul.2008.

[20] Sarıöz K., Kükner A. and Narlı E. Validation of a strip theory based ship motion prediction program. Report No TR 01, Department of Ocean Engineering, Istanbul Technical University, 2000.

[21] Cakici, F., Sukas, O.F., Kinaci, O.K., Alkan, A.D., Linear and Nonlinear Based Ship Motions of a Naval Combatant Model, 9th International Conference on Computational Fluid Dynamics (ICCFD9), Istanbul 2016. 
Ferdi Cakici, Omer Faruk Sukas, Omer Kemal Kinaci, Ahmet Dursun Alkan
Estimation of Vertical Motions of DTMB 5415

Ship Hull with a Fully Nonlinear Approach

[22] A. Olivieri, F. Pistani, A. Avanzini, F.Stern and R. Penna. Towing Tank Experiments Of Resistance, Sinkage and Trim, Boundary Layer, Wake, And Free Surface Flow Around A Naval Combatant Insean 2340 Model, 2001.IIHR Technical Report No. 421

[23] Querard, A.B.G., Temarel, P., Turnock, S.R. (2008). Influence of viscous effects on the hydrodynamics of ship-like sections undergoing symmetric and anti- symmetric motions, using RANS. In: Proceedings of the ASME27th International Conference on Offshore Mechanics and Arctic Engineering (OMAE), Estoril, Portugal, pp.1-10. https://doi.org/10.1115/omae2008-57330.

[24] CD-Adapco, 2014.User guide STAR-CCM Version 9.0.2.

[25] International Towing Tank Conference (ITTC), (2011b). Practical guidelines for ship CFD applications. In: Proceedings of the 26th ITTC.

[26] Stern F, Wilson RV, Coleman HW, Paterson EG (2001). Comprehensive approach to verification and validation of CFD simulations - Part 1: Methodology and procedures. Journal of Fluids Engineering Transactions of the ASME, 123(4), pp. 793-802. https://doi.org/10.1115/1.1412235.

[27] Wilson RV, Stern F, Coleman HW, Paterson EG (2001). Comprehensive approach to verification and validation of CFD simulations - Part 2: Application for RANS simulation of a Cargo/Container ship. Journal of Fluids Engineering - Transactions of the ASME, 123(4), pp. 803-810. https://doi.org/10.1115/1.1412235.

Submitted: 20.05.2016.

Accepted: $\quad 01.02 .2016$.
Ferdi Cakici, fcakici@yildiz.edu.tr

Omer Faruk Sukas

Omer Kemal Kinaci

Ahmet Dursun Alkan

Naval Architecture and Maritime Faculty, Yildiz Technical University, Istanbul, Turkey 